\title{
On the Topology of Simple Fold Maps
}

\author{
Kazuhiro SAKUMA \\ Tokyo Institute of Technology \\ (Communicated by S. Suzuki)
}

\section{§1. Introduction.}

The purpose of this note is to study the topology of simple fold maps of a closed $n$-manifold into a $p$-manifold $(n>p)$ and special generic maps of a closed orientable 4-manifold into an orientable 3-manifold. We call a smooth map $f$ with only fold singularities a fold map and it is said to be simple if the connected component of $f^{-1}(f(x))$ containing $x$ intersects $S(f)$ only at $x$ for every $x \in S(f)$. Special generic maps are smooth maps which have only definite fold sigularities. Precise definitions of fold maps, simple fold maps and special generic maps are given in $\$ 2$.

The results here extend and depend upon some related results in the author's previous paper [13], where special generic maps of simply connected $2 n$-manifolds into $\boldsymbol{R}^{3}$ were studied. For a map $f: M^{n} \rightarrow N^{p}, S(f)$ denotes the set of the singular points of $f$, and when $n-p+1$ is even, $S^{+}(f)$ denotes the set of the fold points with even index and $S^{-}(f)$ the set of the fold points with odd index.

One of our main results is the following

THEOREM A. Let $M^{n}$ be a closed $n$-manifold and $N^{p}$ a $p$-manifold $(n-p$ : odd, $n>p)$. Let $f: M^{n} \rightarrow N^{p}$ be a simple fold map. Then we have

$$
\chi\left(M^{n}\right)=\chi\left(S^{+}(f)\right)-\chi\left(S^{-}(f)\right),
$$

where $\chi$ denotes the Euler characteristic.

When $N^{p}=R^{p}$, the above theorem has been obtained by Fukuda [5] without the assumption that $f$ be simple. It is interesting that the topology of $N^{p}$ does not affect the equality involving the Euler characteristics even if we replace $R^{p}$ by any $p$-manifold in Fukuda's theorem, provided that $f$ is simple.

In [13], for a special generic map $f$ of a simply connected $2 n$-manifold $M^{2 n}$ into $\boldsymbol{R}^{3}$ we have proved that $\chi\left(M^{2 n}\right)=2 \# S(f)$, twice the number of connected components of $S(f)$ (when $2 n=4$, $\# S(f)=\frac{1}{2} b_{2}\left(M^{4}\right)+1$ ). In the case $2 n=4$, if we remove some restrictions on the source and the target manifolds, we have a weaker result on the 
number $\# S(f)$ as follows.

THEOREM B. Let $M^{4}$ be a closed orientable 4-manifold and $N^{3}$ an orientable 3-manifold. Let $f: M^{4} \rightarrow N^{3}$ be a special generic map. Then we have

$$
\# S(f) \leqq \frac{1}{2} b_{2}\left(M^{4}\right)+1 \text {, }
$$

where $\# S(f)$ is the number of connected components of $S(f)$ and $b_{2}\left(M^{4}\right)$ denotes the second betti number of $M^{4}$. Furthermore, the equality holds if $H_{1}\left(M^{4} ; Q\right)=0$.

This theorem for the case $N^{3}=R^{3}$, with a slightly different proof, has been independently proved by Saeki [12].

Moreover, we have several applications of Theorems A and B. One of them is the following

COROllary 4.5. Let $M^{4}$ be a closed 4-manifold with $H_{*}\left(M^{4} ; Q\right) \cong H_{*}\left(\#_{g} S^{1} \times S^{3}\right.$; Q) $(g \geqq 2)$. If $M^{4}$ admits a special generic map $f: M^{4} \rightarrow N^{3}$ into an orientable 3-manifold $N^{3}$, then $S(f)$ is diffeomorphic to the closed orientable surface of genus $g$.

The rest of the paper is organized as follows. In $\$ 2$ we give the definition of the Stein factorization and a proof of Theorem A. In $\$ 3$ we give a proof of Theorem B. $\S 4$ is devoted to examples and applications of Theorems A and B. In $\S 5$ some remarks and related problems will be given.

\section{§2. Stein factorizations and proof of Theorem $\mathbf{A}$.}

Let $f: M^{n} \rightarrow N^{p}(n \geqq p)$ be a smooth map from an $n$-manifold into a $p$-manifold. We denote by $S(f)$ the set of the singularities of $f$. A point $x \in S(f)$ is called a fold singularity if there exist local coordinates $\left(x_{1}, \cdots, x_{n}\right)$ centered at $x$ and $\left(y_{1}, \cdots, y_{p}\right)$ centered at $f(x)$ such that $f$ has the following normal form for some integer $\lambda$ $(0 \leqq \lambda \leqq n-p+1)$ :

$$
\begin{aligned}
& y_{i} \circ f=x_{i} \quad(1 \leqq i \leqq p-1) \\
& y_{p} \circ f=-x_{p}^{2}-\cdots-x_{p+\lambda-1}^{2}+x_{p+\lambda}^{2}+\cdots+x_{n}^{2} .
\end{aligned}
$$

If $\lambda=0$ or $n-p+1$, we call $x \in S(f)$ a definite fold singularity; otherwise, an indefinite fold singularity. If $n-p+1$ is even, the parity of $\lambda$ is independent of the choice of the local coordinates. Hence the two sets $S^{+}(f)=\{x \in S(f) ; \lambda$ is even $\}$ and $S^{-}(f)=\{x \in S(f)$; $\lambda$ is odd $\}$ are well-defined. Note that for a smooth map $f: M^{n} \rightarrow N^{p}(n \geqq p)$ with only fold singularities, $S(f), S^{+}(f)$ and $S^{-}(f)$ are $(p-1)$-dimensional submanifolds of $M^{n}$ and that $f \mid S(f)$ is always an immersion.

Definition 2.1. Let $f: M^{n} \rightarrow N^{p}(n \geqq p)$ be a smooth map with only fold singularities. We call such a map a fold map. If $f$ has no indefinite fold singularities, we call $f$ a special generic map (cf. [2], [9], [12]). A point $x \in S(f)$ is said to be simple if 
the component of $f^{-1}(f(x))$ containing $x$ intersects $S(f)$ only at $x$. Moreover, $f$ is said to be simple if all its singularities are simple (cf. [8]). Note that a special generic map is always simple.

REMARK 2.2. In the above definition, even if $f$ has no singularities, we call $f \dot{a}$ fold map.

DefiNITION 2.3 (Stein factorization, cf. [2][7][8][9][12]). Let $f: M^{n} \rightarrow N^{p}(n>p)$ be a smooth map. We define $W_{f}$ as the quotient space of $M^{n}$ obtained by identifying points of $M^{n}$ which are in the same connected component of a fibre $f^{-1}(y)$ of $f\left(y \in N^{p}\right)$. Let $q_{f}: M^{n} \rightarrow W_{f}$ denote the quotient map and let the map $f^{\prime}: W_{f} \rightarrow N^{p}$ be defined by $f=f^{\prime} \circ q_{f}$. The space $W_{f}$ or the map $q_{f}: M^{n} \rightarrow W_{f}$ is called the Stein factorization of $f$.

REMARK 2.4. Without loss of generality, we may assume that a simple fold map $f: M^{n} \rightarrow N^{p}$ is stable in order to prove Theorem A, where $f$ is stable if every nearby map $g$ in the sense of $C^{\infty}$-topology is identical to $f$, after suitable changes of coordinates, both in the domain and in the range of $g$ (see [6]). For a simple fold map $f: M^{n} \rightarrow N^{p}$ (not necessarily stable), we can modify $f$ so that it becomes a stable map without changing the topologies of $\left(M^{n}, S(f)\right)$ and $\left(M^{n}, S^{ \pm}(f)\right)$ by slightly perturbing $f^{\prime}: W_{f} \rightarrow N^{p}$. This is because we can modify $f^{\prime} \mid q_{f}(S(f))$ so that it becomes an immersion with normal crossings by a small perturbation, since $q_{f} \mid S(f)$ is a homeomorphism. Note that a simple fold map $f: M^{n} \rightarrow N^{p}$ is stable if and only if $f \mid S(f)$ is an immersion with normal crossings (see [6]). Thus, in the following, we assume that all simple fold maps are stable.

In [7], Kushner-Levine-Porto have determined the local structures of $W_{f}$ for a stable map $f$ of a closed 3-manifold into the plane. For a simple fold $\operatorname{map} f: \boldsymbol{M}^{\mathbf{3}} \rightarrow \boldsymbol{R}^{\mathbf{2}}$, the local structures of $W_{f}$ are as in Figure 1 ([8, p. 20]).

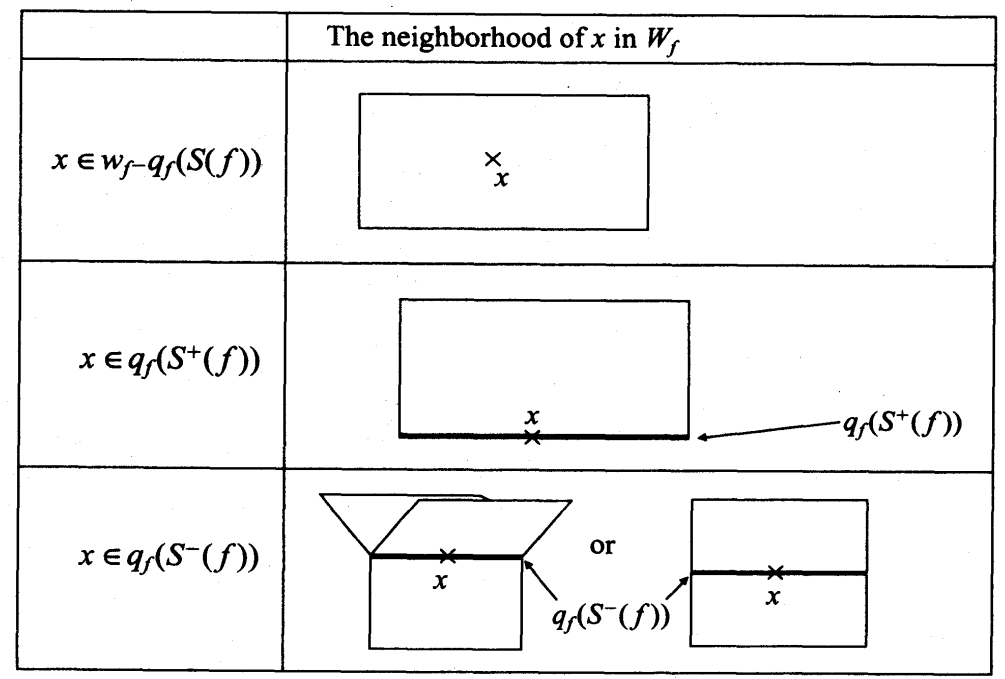

FIGURE 1. 
For a simple fold map $f: M^{n} \rightarrow N^{n-1}$, the local structures of $W_{f}$ are the products of the standard (n-3)-disk $D^{n-3}$ and the conic neighborhoods as in Figure 1.

Now we prove a special case of Theorem A; namely the case $p=n-1$.

THEOREM 2.5. Let $M^{n}$ be a closed n-manifold and $N^{n-1}$ an (n-1)-manifold. Let $f: M^{n} \rightarrow N^{n-1}$ be a simple fold map. Then we have

$$
\chi\left(M^{n}\right)=\chi\left(S^{+}(f)\right)-\chi\left(S^{-}(f)\right) .
$$

Proof. Case (i). $S(f) \neq \varnothing$. We set $\Sigma=q_{f}(S(f))$ and let $N(\Sigma)$ be the regular neighborhood of $\Sigma$ in $W_{f}$. Set $R=W_{f}-\operatorname{Int} N(\Sigma)$. Then we prove the following

ClaIM. $q_{f} \mid q_{f}^{-1}(R): q_{f}^{-1}(R) \rightarrow R$ is an $S^{1}$-bundle over $R$.

(Proof.) Since $f^{\prime} \mid R: R \rightarrow N^{n-1}$ is a local homeomorphism, we can define a smooth structure on $R$ so that $f^{\prime} \mid R$ is an immersion. Then $q_{f} \mid q_{f}^{-1}(R)$ is a proper submersion, since $f \mid M^{n}-S(f)$ is non-singular and $q_{f}^{-1}(R) \subset M^{n}-S(f)$. Thus we have the conclusion by Ehresmann's theorem (cf. [8], [2, Théorème I]). This completes the proof of Claim.

Let $N^{+}(\Sigma)$ (resp. $\left.N^{-}(\Sigma)\right)$ be the regular neighborhood of $q_{f}\left(S^{+}(f)\right)\left(\right.$ resp. $\left.q_{f}\left(S^{-}(f)\right)\right)$ in $W_{f}$. We see that the composition

$$
q_{f}^{-1}\left(N^{+}(\Sigma)\right) \stackrel{q_{f}}{\longrightarrow} N^{+}(\Sigma) \longrightarrow q_{f}\left(S^{+}(f)\right)
$$

is a $D^{2}$-bundle over $q_{f}\left(S^{+}(f)\right)\left(=S^{+}(f)\right)$, where the second map is the bundle projection. Set $S^{-}(f)=S_{1} \cup \cdots \cup S_{m}$, where $S_{j}(1 \leqq j \leqq m)$ are the connected components of $S^{-}(f)$. We set $\Sigma_{j}^{-}=q_{f}\left(S_{j}\right)$ and let $N\left(\Sigma_{j}^{-}\right)$be the regular neighborhood of $\Sigma_{j}^{-}$in $W_{f}(1 \leqq j \leqq m)$. Since $f$ is simple, the composition

$$
q_{f}^{-1}\left(N\left(\Sigma_{j}^{-}\right)\right) \stackrel{q_{f}}{\longrightarrow} N\left(\Sigma_{j}^{-}\right) \longrightarrow q_{f}\left(S_{j}\right)
$$

is a $D_{2}^{2}$ - or $M_{1}^{2}$-bundle over $q_{f}\left(S_{j}\right)\left(=S_{j}\right)(1 \leqq j \leqq m)$, where the second map is the bundle projection, $D_{2}^{2}$ is the 2-disk with two open disks removed and $M_{1}^{2}$ is the Möbius band with an open disk removed (cf. [8]). Thus we have the following decomposition of $M^{n}$ :

$$
M^{n}=q_{f}^{-1}\left(W_{f}\right)=q_{f}^{-1}\left(N^{+}(\Sigma)\right) \cup q_{f}^{-1}(R) \cup \bigcup_{j=1}^{m} q_{f}^{-1}\left(N\left(\Sigma_{j}^{-}\right)\right) \text {. }
$$

Note that $q_{f}^{-1}\left(N^{+}(\Sigma)\right) \cap q_{f}^{-1}\left(N\left(\Sigma_{j}^{-}\right)\right)=\varnothing \quad(1 \leqq j \leqq m)$. Since $q_{f}^{-1}\left(N^{+}(\Sigma)\right) \cap q_{f}^{-1}(R)$ is homeomorphic to an $S^{1}$-bundle over $S^{+}(f)$ and $q_{f}^{-1}\left(N\left(\Sigma_{j}^{-}\right)\right) \cap q_{f}^{-1}(R)$ is homeomorphic to an $\left(S^{1} \cup S^{1} \cup S^{1}\right)$ - or $\left(S^{1} \cup S^{1}\right)$-bundle over $S_{j}(1 \leqq j \leqq m)$, we have

$$
\chi\left(q_{f}^{-1}\left(N^{+}(\Sigma)\right) \cap q_{f}^{-1}(R)\right)=\chi\left(q_{f}^{-1}\left(N\left(\Sigma_{j}^{-1}\right)\right) \cap q_{f}^{-1}(R)\right)=0 \quad(1 \leqq j \leqq m)
$$

by the following well-known facts about the Euler characteristic of a fibration. 
LEMMA 2.6. (1) Let $\pi: E \rightarrow B$ be a fiber bundle with fiber $F$, where $E, B$ and $F$ are finite $C W$-complexes and $\pi$ is cellular. Then we have

$$
\chi(E)=\chi(B) \cdot \chi(F) .
$$

(2) Let $X=A \cup B$ be a finite $C W$-complex and $A, B$ subcomplexes of $X$. Then we have

$$
\chi(A \cup B)=\chi(A)+\chi(B)-\chi(A \cap B) .
$$

Applying this lemma to the decomposition of $M^{n}$, we have

$$
\begin{aligned}
\chi\left(M^{n}\right) & =\chi\left(q_{f}^{-1}\left(N^{+}(\Sigma)\right)\right)+\chi\left(q_{f}^{-1}(R)\right)+\sum_{j=1}^{m} \chi\left(q_{f}^{-1}\left(N\left(\Sigma_{j}^{-}\right)\right)\right) \\
& =\chi\left(S^{+}(f)\right) \cdot \chi\left(D^{2}\right)+\chi(R) \cdot \chi\left(S^{1}\right)+\sum_{j=1}^{m} \chi\left(S_{j}\right) \cdot \chi\left(F_{j}\right),
\end{aligned}
$$

where $F_{j}$ stands for $D_{2}^{2}$ or $M_{1}^{2}$. Then, since $\chi\left(D^{2}\right)=1, \chi\left(S^{1}\right)=0$ and $\chi\left(F_{j}\right)=-1$, we have

$$
\chi\left(M^{n}\right)=\chi\left(S^{+}(f)\right)-\chi\left(S^{-}(f)\right) .
$$

This completes the proof for Case (i).

Case (ii). $S(f)=\varnothing$. In this case, $q_{f}: M^{n} \rightarrow W_{f}$ is an $S^{1}$-bundle over $W_{f}$ (see the proof for the above claim) and hence we have $\chi\left(M^{n}\right)=\chi\left(W_{f}\right) \cdot \chi\left(S^{1}\right)=0$ by Lemma 2.6 (1). Therefore the equality in the statement of the theorem trivially holds. This completes the proof of Theorem 2.5.

We are now in a position to prove Theorem $\mathrm{A}$.

Proof of Theorem A. Case (i). $S(f) \neq \varnothing$. Let $S \subset S(f)$ be a connected component of $S(f)$ and $N(S)$ be the regular neighborhood of $q_{f}(S)$ in $W_{f}$, which is homeomorphic to an $I$-bundle or a $Y$-bundle over $q_{f}(S)$, where $I=[-1,1]$ and $Y=\{r \exp (2 \pi i k / 3) \in C ; 0 \leqq r \leqq 1, k=0,1,2\}$. Then the composition

$$
q_{f}^{-1}(N(S)) \stackrel{q_{f}}{\longrightarrow} N(S) \longrightarrow q_{f}(S)
$$

is a $T$-bundle over $q_{f}(S)$, where the second map is the bundle projection and $T$ is the transverse manifold at a point $x \in S$ (see [8]). More precisely, $T$ is the component of $f^{-1}(\alpha(J))$ containing $x \in S$, where $\alpha: J=[-1,1] \rightarrow N^{p}$ is an embedded arc which passes through $f(x)$ such that $\alpha \bar{T} f$ and $\alpha(J) \cap f(S(f))=\alpha(J) \cap f(S)=\{f(x)\}$. Note that $\operatorname{dim} T=$ $n-p+1$, which is even, and that there exists a Morse function $g: T \rightarrow[-1,1]=J$ which has exactly one critical point whose index is the same as that of $S$ with respect to $f$ (see the definition of the index of $S$ in [11, p. 553]). Hence $\chi(T)=1$ if $x \in S^{+}(f)$ and -1 if $x \in S^{-}(f)$. Therefore the same argument as in the proof of Theorem 2.5 gives the desired formula.

Case (ii). $S(f)=\varnothing$. By the same argument as in the proof of Theorem 2.5, we 
have $\chi\left(M^{n}\right)=0$, since the fiber of the fibration $q_{f}: M^{n} \rightarrow W_{f}$ is of odd dimension and has zero Euler characteristic. Therefore the equality in the statement of the theorem holds. This completes the proof of Theorem A.

We have an immediate corollary of Theorem A.

COROLLARY 2.7. Let $f: M^{n} \rightarrow N^{p}$ be a simple fold map of a closed $n$-manifold into a p-manifold with $n-p+1$ even. Then we have

(i) $\chi\left(M^{n}\right) \equiv \chi(S(f))(\bmod 2)$.

(ii) When $f$ is a special generic map, we have

$$
\chi\left(M^{n}\right)=\chi(S(f)) .
$$

\section{§3. Proof of Theorem B.}

The object of this section is to prove the following

THEOREM B. Let $f: M^{4} \rightarrow N^{3}$ be a special generic map of a closed orientable 4-manifold $M^{4}$ into an orientable 3-manifold $N^{3}$. Then we have

$$
\# S(f) \leqq \frac{1}{2} b_{2}\left(M^{4}\right)+1,
$$

where $\# S(f)$ denotes the number of connected components of $S(f)$ and $b_{2}\left(M^{4}\right)$ is the second betti number of $M^{4}$. The equality holds when $H_{1}\left(M^{4} ; Q\right)=0$.

Before proving Theorem B, we state a lemma which was proved in [12] for the case $N^{3}=R^{3}$. Note that the same proof works also in this general case.

LEMMA 3.1. Let $f: M^{4} \rightarrow N^{3}$ be a special generic map of a closed 4-manifold $M^{4}$ into an orientable 3-manifold $N^{3}$ such that $S(f) \neq \varnothing$ and $q_{f}: M^{4} \rightarrow W_{f}$ its Stein factorization. Then we have the following.

(i) $W_{f}$ is an orientable 3-manifold with boundary.

(ii) $\partial W_{f}$ is homeomorphic to $S(f)$.

(iii) $\left(q_{f}\right)_{*}: \pi_{1}\left(M^{4}\right) \rightarrow \pi_{1}\left(W_{f}\right)$ is an isomorphism.

Proof of Theorem B. Case (i). $S(f) \neq \varnothing$. First we prove the following

ClaIM. $\operatorname{dim}_{\mathbf{Q}} H_{2}\left(W_{f} ; Q\right) \geqq k-1$, where $k=\# S(f)$. Furthermore, if $H_{1}\left(M^{4} ; Q\right)=0$, $\operatorname{dim}_{\mathbf{Q}} H_{2}\left(W_{f} ; Q\right)=k-1$.

(Proof.) The homology exact sequence of the pair $\left(W_{f}, \partial W_{f}\right)$ gives

$0 \rightarrow Q \stackrel{\psi}{\rightarrow} \oplus_{k} Q \stackrel{\phi}{\rightarrow} H_{2}\left(W_{f} ; Q\right) \rightarrow H_{2}\left(W_{f}, \partial W_{f} ; Q\right) \rightarrow H_{1}\left(\partial W_{f} ; Q\right) \rightarrow H_{1}\left(W_{f} ; Q\right) \rightarrow \cdots$,

since $H_{3}\left(W_{f} ; Q\right)=0, H_{3}\left(W_{f}, \partial W_{f} ; Q\right) \cong Q$ and $H_{2}\left(\partial W_{f} ; Q\right) \cong \oplus_{k} Q$. Note that $W_{f}$ is a connected, orientable 3-manifold with boundary consisting of $k$ connected components. 
Hence we have Coker $\psi \cong \bigoplus_{k-1} Q$, since $\psi$ is an injection. Moreover, by the exactness, Coker $\psi=H_{2}\left(\partial W_{f} ; Q\right) / \operatorname{Im} \psi \cong H_{2}\left(\partial W_{f} ; Q\right) / \operatorname{Ker} \phi \cong \operatorname{Im} \phi$. Therefore we have $\operatorname{Im} \phi \cong$ $\oplus_{k-1} Q$ and hence $\operatorname{dim}_{Q} H_{2}\left(W_{f} ; Q\right) \geqq k-1$. From Lemma 3.1 (iii), if $H_{1}\left(M^{4} ; Q\right)=0$, we have $H_{1}\left(W_{f} ; Q\right)=0$ and $H^{1}\left(W_{f} ; Q\right)=0$. By Poincaré-Lefschetz duality, we have $H_{2}\left(W_{f}, \partial W_{f} ; Q\right) \cong H^{1}\left(W_{f} ; Q\right)=0$. Therefore we have $\operatorname{dim}_{Q} H_{2}\left(W_{f} ; Q\right)=k-1$, if $H_{1}\left(M^{4} ; Q\right)=0$. This implies the claim.

Note that by using the above exact sequence together with Lemma 3.1 (ii) we can also show the following

LEMMA 3.2. If $H_{1}\left(M^{4} ; Q\right)=0, S(f)$ is homeomorphic to a disjoint union of 2-spheres.

Lemma 3.2 will be used in $\S 4$.

Now we take the double of $W_{f}$ and set $X=W_{f} \cup_{\partial W_{f}} W_{f}$. Then $X$ is a closed orientable 3-manifold. By Lemma 3.1 (ii) and Lemma 2.6 (2),

$$
0=\chi(X)=2 \chi\left(W_{f}\right)-\chi\left(\partial W_{f}\right)=2 \chi\left(W_{f}\right)-\chi(S(f)) .
$$

Combining Corollary 2.7 (ii) with Lẹma 3.1 (iii), we have

$$
\begin{aligned}
\chi\left(M^{4}\right) & =\chi(S(f))=2 \chi\left(W_{f}\right) \\
& =2\left(1-b_{1}\left(W_{f}\right)+b_{2}\left(W_{f}\right)\right) \\
& =2\left(1-b_{1}\left(M^{4}\right)+b_{2}\left(W_{f}\right)\right) \\
& \geqq 2\left(1-b_{1}\left(M^{4}\right)+k-1\right)=2\left(\# S(f)-b_{1}\left(M^{4}\right)\right),
\end{aligned}
$$

where $b_{i}$ denotes the $i$-th betti number. On the other hand, by Poincaré duality we have

$$
\chi\left(M^{4}\right)=2\left(1-b_{1}\left(M^{4}\right)\right)+b_{2}\left(M^{4}\right) .
$$

The inequality in the statement of the theorem follows formally from $(*)$ and $(* *)$ and the equality holds when $H_{1}\left(M^{4} ; Q\right)=0$. This completes the proof for Case (i).

Case (ii). $S(f)=\varnothing$. The inequality in the statement of the theorem trivially follows. Moreover, in this case $q_{f}: M^{4} \rightarrow W_{f}$ is an $S^{1}$-bundle over $W_{f}$ (see the proof for the claim in the proof of Theorem 2.5) and hence $\chi\left(M^{4}\right)=0$ by Lemma 2.6 (1). On the other hand, if $H_{1}\left(M^{4} ; Q\right)=0$, then $H_{3}\left(M^{4} ; Q\right)=0$ and $\chi\left(M^{4}\right) \neq 0$. This means that $S(f) \neq \varnothing$ if $H_{1}\left(M^{4} ; Q\right)=0$. Therefore this case does not occur if $H_{1}\left(M^{4} ; Q\right)=0$. This completes the proof of Theorem B.

COROLlary 3.3. Let $M^{4}$ be a closed orientable 4-manifold with odd Euler characteristic. Then $M^{4}$ does not admit any special generic map into an orientable 3-manifold.

Proof. If $M^{4}$ admits a special generic map into an orientable 3-manifold, we have $\chi\left(M^{4}\right)=\chi(S(f))=2 \chi\left(W_{f}\right) \equiv 0(\bmod 2)$ (see the proof of Theorem B), if $S(f) \neq \varnothing$. 
Recall that, if $S(f)=\varnothing, \chi\left(M^{4}\right)$ must vanish. This completes the proof.

\section{§4. Applications.}

In this section we give some applications of Theorems A and B to the study of special generic maps of closed orientable 4-manifolds into orientable 3-manifolds.

LEMMA 4.1. Let $M^{4}$ be a closed 4-manifold with non-zero Euler characteristic. Then $M^{4}$ does not admit a smooth map $f$ into an orientable 3-manifold such that $S(f)=\varnothing$.

This follows from the same argument as in the proof for Case (ii) in the proof of Theorem B.

COROLlaRY 4.2. Let $M^{4}$ be a closed simply connected 4-manifold and $N^{3}$ an orientable 3-manifold. If $M^{4}$ admits a special generic map $f: M^{4} \rightarrow N^{3}$ such that $S(f)$ is connected, then $\mathrm{M}^{4}$ is homeomorphic to $\mathrm{S}^{4}$, the 4-sphere.

Proof. Since $M^{4}$ is simply connected, $\chi\left(M^{4}\right) \neq 0$. By Lemma 4.1 we have $S(f) \neq \varnothing$. Moreover, by Lemma 3.2, $S(f)$ is diffeomorphic to the 2-sphere. By Corollary 2.7 (ii) $M^{4}$ is a homotopy 4-sphere, which is homeomorphic to $S^{4}$ (see [4]).

COROLLARY 4.3. Let $f: S^{4} \rightarrow N^{3}$ be a special generic map of the standard 4-sphere into an orientable 3-manifold $N^{3}$. Then $S(f)$ is diffeomorphic to the 2-sphere and is topologically unknotted in $S^{4}$.

Proof. Since $\chi\left(S^{4}\right) \neq 0, S(f) \neq \varnothing$ by Lemma 4.1. By Theorem B we have $\# S(f)=1$. Moreover, by Lemma $3.2 S(f)$ is diffeomorphic to the 2-sphere. We use the same notations as in the proof of Theorem 2.5. Then $S^{4}$ is decomposed as follows:

$$
S^{4}=q_{f}^{-1}\left(N^{+}(\Sigma)\right) \cup q_{f}^{-1}(R) .
$$

Note that $W_{f}$ is homeomorphic to $R$ and that $S^{4}-S(f)$ is homotopy equivalent to $q_{f}^{-1}(R)$. Recall that $q_{f}^{-1}(R)$ is diffeomorphic to an $S^{1}$-bundle over $W_{f}$. On the other hand, since $S(f) \cong S^{2}$ and $\pi_{1}\left(W_{f}\right) \cong \pi_{1}\left(S^{4}\right)=1$ by Lemma 3.1 (iii), $W_{f}$ is a homotopy 3-ball. Hence $q_{f}^{-1}(R)$ is diffeomorphic to $W_{f} \times S^{1}$. Hence we have

$$
\pi_{1}\left(S^{4}-S(f)\right) \cong \pi_{1}\left(W_{f} \times S^{1}\right) \cong \pi_{1}\left(S^{1}\right) \cong Z \text {. }
$$

Applying Freedman's result ([4]), we obtain the conclusion.

Next we give fundamental examples of special generic maps of 4-manifolds into orientable 3-manifolds such that every orientable closed surface is realized as the singular set. Let $M^{4}$ be a closed 4-manifold with $H_{*}\left(M^{4} ; Q\right) \cong H_{*}\left(S^{1} \times S^{3} ; Q\right)$. Now suppose that $M^{4}$ admits a special generic map $f: M^{4} \rightarrow N^{3}$ such that $S(f) \neq \varnothing$ for some orientable 3-manifold $N^{3}$. Note that $\chi\left(M^{4}\right)=\chi\left(S^{1} \times S^{3}\right)=0$ and $b_{2}\left(M^{4}\right)=b_{2}\left(S^{1} \times S^{3}\right)=0$. By Theorems $\mathrm{A}$ and $\mathrm{B}$, we have $\chi(S(f))=0$ and $\sharp S(f) \leqq 1$. Moreover, $S(f)$ is orientable (see Lemma 3.1). Thus we obtain 
COROllary 4.4. Let $M^{4}$ be a closed 4-manifold with $H_{*}\left(M^{4} ; Q\right) \cong H_{*}\left(S^{1} \times S^{3}\right.$; Q). If $M^{4}$ admits a special generic map $f: M^{4} \rightarrow N^{3}$ into an orientable 3-manifold $N^{3}$ such that $S(f) \neq \varnothing$, then $S(f)$ is diffeomorphic to the torus.

In fact, we can construct a special generic map of $S^{1} \times S^{3}$ into $R^{3}$ as in Corollary 4.4 as follows. Let $\psi_{1}: S^{3} \rightarrow R^{2}$ be the special generic map defined by $\psi_{1}=\pi \mid S^{3}$, where $\pi: \boldsymbol{R}^{4} \rightarrow \boldsymbol{R}^{2}$ is the standard projection defined by $\pi(x, y, z, w)=(x, y)$. Let $\omega: S^{1} \times \boldsymbol{R}^{2} \rightarrow$ $\boldsymbol{R}^{3}$ be a standard embedding and define $\psi: S^{1} \times S^{3} \rightarrow S^{1} \times R^{2}$ by $\psi\left(x_{1}, x_{2}\right)=\left(x_{1}, \psi_{1}\left(x_{2}\right)\right)$ for $x_{1} \in S^{1}, x_{2} \in S^{3}$. Then the composite map $f: \omega \circ \psi$ is the required one. Clearly, $S(f)$ is diffeomorphic to the torus. Moreover, by connected summing $S^{1} \times S^{3}$ 's, we obtain a special generic map of $\#_{g} S^{1} \times S^{3}(g \geqq 2)$ into $R^{3}$ such that the singular set is diffeomorphic to $\Sigma_{g}$, the closed orientable surface of genus $g$. Such a construction can be found in [12, Lemma 5.4].

COROLlaRY 4.5. Let $M^{4}$ be a closed 4-manifold with $H_{*}\left(M^{4} ; Q\right) \cong H_{*}\left(\#_{g} S^{1} \times S^{3}\right.$; Q) $(g \geqq 2)$. If $M^{4}$ admits a special generic map $f: M^{4} \rightarrow N^{3}$ into an orientable 3-manifold $N^{3}$, then $S(f)$ is diffeomorphic to $\Sigma_{g}$, the closed orientable surface of genus $g$.

This follows by a similar argument as in the proof of Corollary 4.4. Note that $S(f) \neq \varnothing$ by Lemma 4.1 .

We have seen in Corollary 4.3 that if $f: S^{4} \rightarrow N^{3}$ is a special generic map into an orientable 3-manifold $N^{3}$, then $S(f)$ is diffeomorphic to the 2-sphere, which is topologically unknotted. For fold maps, the situation is much more complicated; however, we can prove the following, for example.

Proposition 4.6. Let $\Sigma^{4}$ be a closed 4-manifold with $H_{*}\left(\Sigma^{4} ; Z\right) \cong H_{*}\left(S^{4} ; Z\right)$ and $f: \Sigma^{4} \rightarrow \boldsymbol{R}^{3}$ a fold map. Then $S(f)$ contains no closed non-orientable surface of odd genus.

Proof. Suppose that $S(f)$ contains a closed non-orientable surface $F$ of odd genus. By [11], it is contained in $S^{-}(f)$ and its self-intersection number is zero. On the other hand, by generalized Whitney's congruence $[10]$, we have $F \cdot F \equiv 2 \chi(F)(\bmod 4)$, since the signature of $\Sigma^{4}$ vanishes. Hence we have $F \cdot F \neq 0$. This means that $F \not S^{-}(f)$, which is a contradiction.

This proposition and the question (see Problem (4) in the next section) were pointed out to the author by $\mathrm{O}$. Saeki.

REMARK 4.7. By Eliasberg [3], a closed 4-manifold $\Sigma^{4}$ with $H_{*}\left(\Sigma^{4} ; Z\right) \cong H_{*}\left(S^{4}\right.$; $Z$ ) always admits a fold map into $R^{3}$, since $\Sigma^{4}$ is stably parallelizable.

\section{§5. Problems and concluding remarks.}

In conclusion we summarize several problems which have been suggested by the results of this note. 
(1) In Corollary 4.2 is $M^{4}$ diffeomorphic to $S^{4}$ ?

(2) In Corollary 4.3 is $S(f)$ smoothly unknotted in $S^{4}$ ?

(3) Does a closed orientable 4-manifold with odd Euler characteristic admit a fold map into an orientable 3-manifold? (cf. Corollary 3.3.)

(4) Does a closed 4-manifold $\Sigma^{4}$ with $H_{*}\left(\Sigma^{4} ; Z\right) \cong H_{*}\left(S^{4} ; Z\right)$ admit a fold map into $\boldsymbol{R}^{3}$ such that $S(f)$ contains a non-orientable surface (of even genus)? (cf. Corollary 4.6.)

Problems (1) and (2) are extremely difficult. Problem (3) is an interesting problem in singularity theory. Singularities of a stable map of an $n$-manifold $(n \geqq 3)$ into a 3-manifold are fold singularities, cusp singularities and swallowtails. They cannot be eliminated by small perturbations. It is known ([1]) that a smooth map of a closed orientable 4-manifold $M^{4}$ into an orientable 3-manifold is homotopic to a stable map without swallowtails. By Corollary 3.3 we have that every stable map of a closed orientable 4-manifold $M^{4}$ with $\chi\left(M^{4}\right)$ odd into an orientable 3-manifold has indefinite fold singularities. However, we do not know whether cusp singularities can be eliminated for a stable map of a closed orientable 4-manifold $M^{4}$ into an orientable 3-manifold without swallowtails, even if we suppose that $\chi\left(M^{4}\right)$ is odd. In fact, the Thom polynomial for cusp singularities vanishes (cf. [1]). This is because $\left[\overline{A_{2}(f)}\right]_{2}^{*}$ should be of the form $a w_{1}^{3}+b w_{1} w_{2}+c w_{3}\left(a, b, c \in Z_{2}\right)$ and $w_{1}=0, w_{3}=0($ see $[14, \S 23])$, where $A_{2}(f)$ denotes the set of the cusp singularities of $f: M^{4} \rightarrow R^{3}$ (see [5]) and $\left[\overline{A_{2}(f)}\right]_{2}^{*} \in H^{3}\left(M^{4} ; Z_{2}\right)$ is the Poincare dual of the $Z_{2}$-homology class $\left[\overline{A_{2}(f)}\right]_{2}$ represented by the closure of $A_{2}(f)$. Thus the Thom polynomial for cusp singularities has no information about this question. It is recently proved as a partial answer to this question by Saeki ([11, Theorem 4]) that a closed 4-manifold $M^{4}$ with $H_{*}\left(M^{4} ; Z\right) \cong H_{*}\left(C P^{2} ; Z\right)$ admits no fold map into $\boldsymbol{R}^{3}$. In [11], it is also proved that a closed orientable 4-manifold $M^{4}$ with $\chi\left(M^{4}\right)$ odd never admits a simple fold map into $\boldsymbol{R}^{3}$.

Acknowledgement. The author wishes to thank Prof. Osamu Saeki for many valuable discussions and helpful comments. He also thanks the referee for pointing out some mistakes in an earlier version of the manuscript.

\section{References}

[ 1 ] Y. ANDO, On the higher Thom polynomials of Morin singularities, Publ. RIMS Kyoto Univ. 23 (1987), 195-207.

[ 2] O. BURLET and G. DE RHAM, Sur certaines applications génériques d'une variété close à 3-dimensions dans le plan, L'Enseign. Math. 20 (1974), 275-292.

[ 3 ] J. M. ElIASBERG, Surgery of singularities of smooth mappings, Math. USSR Izv. 4(1972), 1302-1326.

[ 4 ] M. H. FreEdMaN, The topology of four dimensional manifolds, J. Differential Geom. 17(1982), $357-453$.

[ 5 ] T. FukUDA, Topology of folds, cusps and Morin singularities, A Fete of Topology (ed. by Y. Matsumoto, T. Mizutani and S. Morita), Academic Press (1987), 331-353.

[6] M. Golubitsky and V. Gulllemin, Stable Mappings and Their Singularities, Graduate Texts in Math. 
14 (1973), Springer.

[7] L. Kushner, H. Levine and P. Porto, Mapping three manifolds into the plane, Bol. Soc. Mat. Mexicana 29 (1984), 11-34.

[8] H. Levine, Classifying Immersions into $\boldsymbol{R}^{\mathbf{4}}$ over Stable Maps of 3-Manifolds into $\boldsymbol{R}^{2}$, Lecture Notes in Math. 1157 (1985), Springer.

[9] P. Porto and Y. K. S. FuruYa, On special generic maps from a closed manifold into the plane, Topology Appl. 35 (1990), 41-52.

[10] V. Rohlin, Proof of Gudkov's hypothesis, Funct. Anal. 6 (1972), 62-64 (English translation, 136-138).

[11] O. SAEKi, Notes on the topology of folds, J. Math. Soc. Japan 44 (1992), 551-566.

[12] - Topology of special generic maps of manifolds into Euclidean spaces, Topology Appl. 49 (1993), 265-293.

[13] K. Sakuma, On special generic maps of simply connected $2 n$-manifolds into $\boldsymbol{R}^{3}$, Topology Appl. 50 (1993), 249-261.

[14] H. Whitney, On the Topology of Differentiable Manifolds, Lectures in Topology, Univ. Michigan Press (1941).

Present Address:

Department of General Education, Kochi National College of Technology, MONOBE 200-1, KOCHI, 783 JAPAN. 
<smiles>[CH]</smiles> 